\title{
Medical Image Fusion via an Effective Wavelet-Based Approach
}

\author{
Yong Yang, ${ }^{1,2,3}$ Dong Sun Park, ${ }^{2}$ Shuying Huang, ${ }^{4}$ and Nini Rao ${ }^{3}$ \\ ${ }^{1}$ School of Information Technology, Jiangxi University of Finance and Economics, Nanchang, Jiangxi 330013, China \\ ${ }^{2}$ Department of Electronics and Information Engineering, Chonbuk National University, Jeonju, Jeonbuk 561-756, Republic of Korea \\ ${ }^{3}$ School of Life Science and Technology, University of Electronic Science and Technology of China, Chengdu 610054, China \\ ${ }^{4}$ School of Software and Communications Engineering, Jiangxi University of Finance and Economics, Nanchang, Jiangxi 330013, China
}

Correspondence should be addressed to Yong Yang, greatyang@foxmail.com

Received 30 July 2009; Revised 27 October 2009; Accepted 10 March 2010

Academic Editor: A. Enis Cetin

Copyright ( $) 2010$ Yong Yang et al. This is an open access article distributed under the Creative Commons Attribution License, which permits unrestricted use, distribution, and reproduction in any medium, provided the original work is properly cited.

A novel wavelet-based approach for medical image fusion is presented, which is developed by taking into not only account the characteristics of human visual system (HVS) but also the physical meaning of the wavelet coefficients. After the medical images to be fused are decomposed by the wavelet transform, different-fusion schemes for combining the coefficients are proposed: coefficients in low-frequency band are selected with a visibility-based scheme, and coefficients in high-frequency bands are selected with a variance based method. To overcome the presence of noise and guarantee the homogeneity of the fused image, all the coefficients are subsequently performed by a window-based consistency verification process. The fused image is finally constructed by the inverse wavelet transform with all composite coefficients. To quantitatively evaluate and prove the performance of the proposed method, series of experiments and comparisons with some existing fusion methods are carried out in the paper. Experimental results on simulated and real medical images indicate that the proposed method is effective and can get satisfactory fusion results.

\section{Introduction}

Nowadays, with the rapid development in high-technology and modern instrumentations, medical imaging has become a vital component of a large number of applications, including diagnosis, research, and treatment. In order to support more accurate clinical information for physicians to deal with medical diagnosis and evaluation, multimodality medical images are needed, such as X-ray, computed tomography $(\mathrm{CT})$, magnetic resonance imaging (MRI), magnetic resonance angiography (MRA), and positron emission tomography (PET) images [1]. These multimodality medical images usually provide complementary and occasionally conflicting information. For example, the CT image can provide dense structures like bones and implants with less distortion, but it cannot detect physiological changes, while the MR image can provide normal and pathological soft tissues information, but it cannot support the bones information. In this case, only one kind of image may not be sufficient to provide accurate clinical requirements for the physicians. Therefore, the fusion of the multimodal medical images is necessary and it has become a promising and very challenging research area in recent years $[2,3]$.

Image fusion can be broadly defined as the process of combing multiple input images or some of their features into a single image without the introduction of distortion or loss of information [4]. The aim of image fusion is to integrate complementary as well as redundant information from multiple images to create a fused image output. Therefore, the new image generated should contain a more accurate description of the scene than any of the individual source images and is more suitable for human visual and machine perception or further image processing and analysis tasks [5]. For medical image fusion, the fusion of images can often lead to additional clinical information not apparent in the separate images. Another advantage is that it can reduce the storage cost by storing just the single fused image instead of multisource images.

So far, many techniques for image fusion have been proposed in the literature and a thorough overview of 
these methods can be viewed in [6]. According to the stage at which the combination mechanism takes place, the image fusion methods can be generally grouped into three categories, namely, pixel level or sensor level, feature level, and decision level [7]. Since the pixel level fusion has the advantage that the images used contain the original measured quantities, and the algorithms are computationally efficient and easy to implement, the most image fusion applications employ pixel level-based methods [8]. Therefore, in this paper, we are still concerned about pixel level fusion, and when the terms "image fusion" or "fusion" are used, pixellevel fusion is intended.

The simplest way of image fusion is to take the average of the two images pixel by pixel. However, this method usually leads to undesirable side effect such as reduced contrast [9]. More robust algorithm for pixel level fusion is the weighted average approach. In this method, the fused pixel is estimated as the weighted average of the corresponding input pixels. However, the weight estimation usually requires a user-specific threshold. Other methods have been developed, such as intensity-hue-saturation (IHS), principal component analysis (PCA), and the Brovey transform [10]. These techniques are easy to understand and implement. However, although the fused images obtained by these methods have high spatial quality, they usually suffer from spectral degradation; that is, they can yield high spatial resolutionfused image, but they overlook the high quality of spectral information which is especially crucial for remote sensing image fusion [10]. Artificial neural network (ANN) has also been introduced to make image fusion, as seen in [11]. However, the performance of ANN depends on the sample images and this is not an appealing characteristic. Yang et al. used a statistical approach to fuse the images [12]; however, in his method the distortion is modeled as a mixture of Gaussian probability density functions (pdfs) which is a limiting assumption. Because the real-world objects usually contain structures at many different scales or resolutions and mutilresolution or multiscale approaches can provide a means to exploit this fact, the multiresolution techniques have then attracted more and more interest in image fusion.

The multiresolution techniques involve two kinds, one is pyramid transform; another is wavelet transform. In the pyramid fusion, the input images are first transformed into their multiresolution pyramid representations. The fusion process then creates a new fused pyramid from the input image pyramids in a certain fusion rule. The fused image is finally reconstructed by performing an inverse multiresolution transform. Examples of this approach include the Laplacian pyramid [13], the gradient pyramid [14], the contrast pyramid [15], the ratio-of-low-pass pyramid [16], and the morphological pyramid [17]. However, for the reason of the pyramid method fails to introduce any spatial orientation selectivity in the decomposition process, the above mentioned methods often cause blocking effects in the fusion results [18]. Matsopoulos et al. earlier applied the morphological pyramid method to fuse the MR and CT images [19], but this method can occasionally create many undesired edges. Another family of the multiresolution fusion techniques is the wavelet-based method, which usually used the discrete wavelet transform (DWT) in the fusion. Since the DWT of image signals produces a nonredundant image representation, it can provide better spatial and spectral localization of image information as compared to other multiresolution representations. The research results reveal that DWT schemes have some advantages over pyramid schemes such as increased directional information, no blocking artifacts that often occur in pyramid fused images; better signal-to-noise ratios [11]. Therefore, the waveletbased method has been popular widely used for image fusion $[5,18,20-23]$, and two detailed surveys can be seen in $[24,25]$. Although there are considerable wavelet-based fusion works today, most of them concerned on remote images, multifocus images, and infrared images, while less work has been done for medical images. Yu et al. fused the medical images by the wavelet-based method with a maximum-selection fusion rule [26], which is similar to Burt's method [14]. However, this method suffers from the noise and artifacts as they tend to have higher contrast. $\mathrm{Qu}$ et al. used the modulus maxima selection criteria for the wavelet transform coefficients in the medical image fusion [27]. The disadvantage of this method is that they consider only wavelet coefficients (pixel) values while making decisions about constructing the fused image [28]. More recently, Cheng et al. proposed a weighted wavelet-based method for fusion of PET and CT images [29]. However, their method confronted with the problem of selecting the parameters of weight; that is to say their method depended on the weights given by the user. Therefore, different weights will lead to different fused results.

In this paper, a novel and fully automated wavelet-based method for medical image fusion is proposed. The main contribution of this work is that after the source images are decomposed by the wavelet transform, the coefficients of the low-frequency portion and high-frequency portions are performed with different fusion schemes. This new technique is developed by not only taking into account the characteristics of the human visual system (HVS) for the wavelet coefficients but also considering the physical meaning of the coefficients. Therefore, the coefficients of the low-frequency and high-frequency bands are treated with different ways: the former is selected with a visibility based scheme, and the latter is selected by a maximum local variance scheme. Besides, in order to avoid the presence of noise and guarantee the homogeneity of the fused image, all the coefficients are finally performed with a consistency verification. The fused image can then be achieved by an inverse wavelet transform with the coefficients obtained from all frequency bands. Both qualitative and quantitative performance evaluations are made and verified in the paper.

The remainder of the paper is organized as follows. The related wavelet-based image fusion technique is reviewed and given in Section 2. The proposed method for fusing multimodal medical images is described in Section 3. Experimental results and analysis are presented in Section 4 and the conclusions are given in Section 5 . 


\section{Image Fusion Based on Wavelet Transform}

The original concept and theory of wavelet-based multiresolution analysis came from Mallat [30]. The wavelet transform is a mathematical tool that can detect local features in a signal process. It also can be used to decompose twodimensional (2D) signals such as 2D gray-scale image signals into different resolution levels for multiresolution analysis. Wavelet transform has been greatly used in many areas, such as texture analysis, data compression, feature detection, and image fusion. In this section, we briefly review and analyze the wavelet-based image fusion technique.

2.1. Wavelet Transform. Wavelet transforms provide a framework in which a signal is decomposed, with each level corresponding to a coarser resolution or lower-frequency band and higher-frequency bands. There are two main groups of transforms, continuous and discrete. Of particular interest is the DWT, which applies a two-channel filter bank (with downsampling) iteratively to the lowpass band (initially the original signal). The wavelet representation then consists of the low-pass band at the lowest resolution and the highpass bands obtained at each step. This transform is invertible and nonredundant.

The DWT is a spatial-frequency decomposition that provides a flexible multiresolution analysis of an image [31]. In one dimension (1D) the basic idea of the DWT is to represent the signal as a superposition of wavelets. Suppose that a discrete signal is represented by $f(t)$; the wavelet decomposition is then defined as

$$
f(t)=\sum_{m, n} c_{m, n} \psi_{m, n}(t),
$$

where $\psi_{m, n}(t)=2^{-m / 2} \psi\left[2^{-m} t-n\right]$ and $m$ and $n$ are integers. There exist very special choices of $\psi$ such that $\psi_{m, n}(t)$ constitutes an orthonormal basis, so that the wavelet transform coefficients can be obtained by an inner calculation:

$$
c_{m, n}=\left\langle f, \psi_{m, n}\right\rangle=\int \psi_{m, n}(t) f(t) d t .
$$

In order to develop a multiresolution analysis, a scaling function $\phi$ is needed, together with the dilated and translated version of it, $\phi_{m, n}(t)=2^{-m / 2} \phi\left[2^{-m} t-n\right]$. According to the characteristics of the scale spaces spanned by $\phi$ and $\psi$, the signal $f(t)$ can be decomposed in its coarse part and details of various sizes by projecting it onto the corresponding spaces.

Therefore, to find such decomposition explicitly, additional coefficients $a_{m, n}$ are required at each scale. At each scale $a_{m, n}$ and $a_{m-1, n}$ describe the approximations of the function $f$ at resolution $2^{m}$ and at the coarser resolution $2^{m-1}$, respectively, while the coefficients $c_{m, n}$ describe the information loss when going from one approximation to another. In order to obtain the coefficients $c_{m, n}$ and $a_{m, n}$ at each scale and position, a scaling function is needed that is similarly defined to (2). The approximation coefficients and wavelet coefficients can be obtained:

$$
a_{m, n}=\sum_{k} h_{2 n-k} a_{m-1, k}
$$

$$
c_{m, n}=\sum_{k} g_{2 n-k} a_{m-1, k}
$$

where $h_{n}$ is a lowpass FIR filter and $g_{n}$ is related highpass FIR filter. To reconstruct the original signal the analysis filters can be selected from a biorthogonal set which have a related set of synthesis filters. These synthesis filters $\tilde{h}$ and $\tilde{g}$ can be used to perfectly reconstruct the signal using the reconstruction formula:

$$
a_{m-1, l}(f)=\sum_{n}\left[\tilde{h}_{2 n-l} a_{m, n}(f)+\tilde{g}_{2 n-l} c_{m, n}(f)\right] .
$$

Equations (3) and (4) are implemented by filtering and downsampling. Conversely (5) is implemented by an initial upsampling and a subsequent filtering.

In a 2-D DWT, a 1-D DWT is first performed on the rows and then columns of the data by separately filtering and downsampling. This results in one set of approximation coefficients $I_{a}$ and three sets of detail coefficients, as shown in Figure $1(\mathrm{a})$, where $I_{b}, I_{c}$, and $I_{d}$ represent the horizontal, vertical and dialog directions of the image $I$, respectively. In the language of filter theory, these four subimages correspond to the outputs of low-low (LL), lowhigh (LH), high-low (HL), and high-high (HH) bands. By recursively applying the same scheme to the LL subband a multiresolution decomposition with a desires level can then be achieved. Therefore, a DWT with K decomposition levels will have $M=3 * K+1$ such frequency bands. Figure 1(b) shows the 2-D structures of the wavelet transform with two decomposition levels. It should be noted that for a transform with $K$ levels of decomposition, there is always only one lowfrequency band ( $L L^{K}$ in Figure 1(b)); the rest of bands are high-frequency bands in a given decomposition level.

2.2. Fusion with Wavelet Transform. In this subsection, to better understand the concept and procedure of the waveletbased fusion technique, a schematic diagram is first given in Figure 2. In general, the basic idea of image fusion based on wavelet transform is to perform a multiresolution decomposition on each source image; the coefficients of both the low-frequency band and high-frequency bands are then performed with a certain fusion rule as displayed in the middle block of Figure 2 The widely used fusion rule is maximum selection scheme. This simple scheme just selects the largest absolute wavelet coefficient at each location from the input images as the coefficient at the location in the fused image. After that, the fused image is obtained by performing the inverse DWT (IDWT) for the corresponding combined wavelet coefficients. Therefore, as shown in Figure 2, the detailed fusion steps based on wavelet transform can be summarized below.

Step 1. The images to be fused must be registered to assure that the corresponding pixels are aligned.

Step 2. These images are decomposed into wavelet transformed images, respectively, based on wavelet transformation. The transformed images with $K$-level decomposition will include one low-frequency portion (low-low band) 


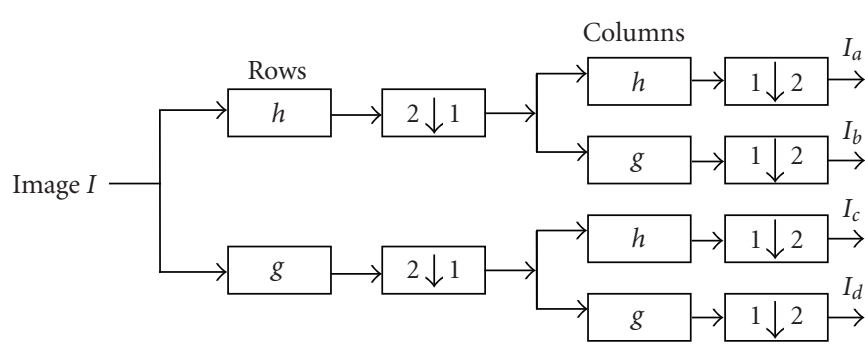

(a)

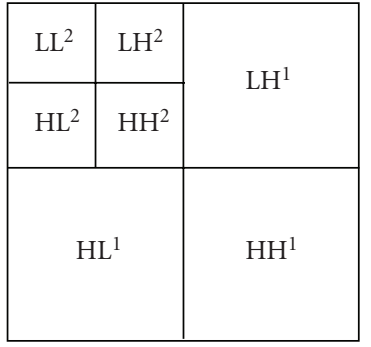

(b)

FIGURE 1: Structures of 2-D DWT. (a) One stage of 2-D DWT multiresolution image decomposition; (b) 2-D DWT structure with labeled subbands in two-level decomposition.

and $3 K$ high-frequency portions (low-high bands, high-low bands, and high-high bands).

Step 3. The transform coefficients of different portions or bands are performed with a certain fusion rule.

Step 4. The fused image is constructed by performing an inverse wavelet transform based on the combined transform coefficients from Step 3.

\section{The Proposed Fusion Method}

As shown in the fusion block, Figure 2, it is easy to find that the core step in image fusion based on wavelet is that of coefficient combination, namely, the fusion rule because it will decide how to merge the coefficients in an appropriate way so that a high-quality fused image can be obtained. Therefore, for this kind of image fusion method the key issue is its fusion rule design, and it should be paid more attention. Over the past years, various fusion rules have been proposed, which can be divided into pixel-based method and window-based method. The popular widely used pixelbased fusion rule is the aforementioned maximum selection scheme [20]. This method can select the salient features from the source images; however, it is sensitive to noise and artifacts as they intend to have higher contrast. As a result, with this method some noise and artifacts are easily introduced into the fused image, which will reduce the resultant image quality consequently. Averaging fusion rule is another pixel-based method and it can lead to a stabilization of the fusion result. However, this scheme tends to blur images and reduce the contrast of features appearing in only one image. More complex fusion rules such as window-based or region-based are also proposed because these types of schemes are more robust than the pixelbased scheme against the image misregistration. Burt and Kolczynshi [14] proposed a window-based weighted average fusion rule. However, the weights in this scheme rely on a user predefined threshold. Li et al. [18] used an areabased maximum selection rule to determine which of the input is likely to contain the most useful information by considering the maximum absolute variance value of the central coefficients within a window. Although this method has been proved better than the pyramid-based method, the disadvantage of this method is that it treats the wavelet coefficients of both low-frequency band and high-frequency bands in the same way. However, as we know in many applications, the ultimate user or interpreter of the fused image is a human. So the human perception should be considered in the image fusion. According to the theoretical models of the HVS, it is easy to know that the human eyes have different sensitiveness to the wavelet coefficients of low resolution band and high resolution bands [32, 33]. Hence, the above fusion rules that treat all the coefficients in same way will have some disadvantages.

On the other hand, since the main objective of this paper is to fuse the multimodal medical images, the characteristics of the images should also be considered. Figure 3 illustrates an example of the original CT and MR images. From Figure 3 it is easy to see that the CT image provides clear bones information but no soft tissues information, while contrast to CT image the MR image provides clear soft tissues information but no bones information. That is to say, the same object in the two medical images appears very distinctly. Hence, when the two images are decomposed by wavelet transform, the approximation image (low-frequency band) and the detail image (high-frequency bands) may have very different physical meaning. Based on this and the above analysis, this paper presents a new fusion rule to perform the wavelet coefficients which treats the lowfrequency band and high-frequency bands with different fusion schemes separately. The coefficients of low-frequency band are selected by a visibility based selection scheme, while the coefficients of the high-frequency bands are performed with a maximum window-based variance selection scheme. Then in order to overcome the influence of the noise and guarantee the homogeneity of the fused image a windowbased consistency verification is employed to all the coefficients selected from all the frequency bands. The overall flowchart of our proposed fusion rule can be depicted as in Figure 4.

3.1. Low-Frequency Band Fusion. In this paper, to simplify the description of the different alternatives available in forming a fusion rule, as in $[5,24]$ we also consider only two source images, $X$ and $Y$, and the fused image $Z$. 


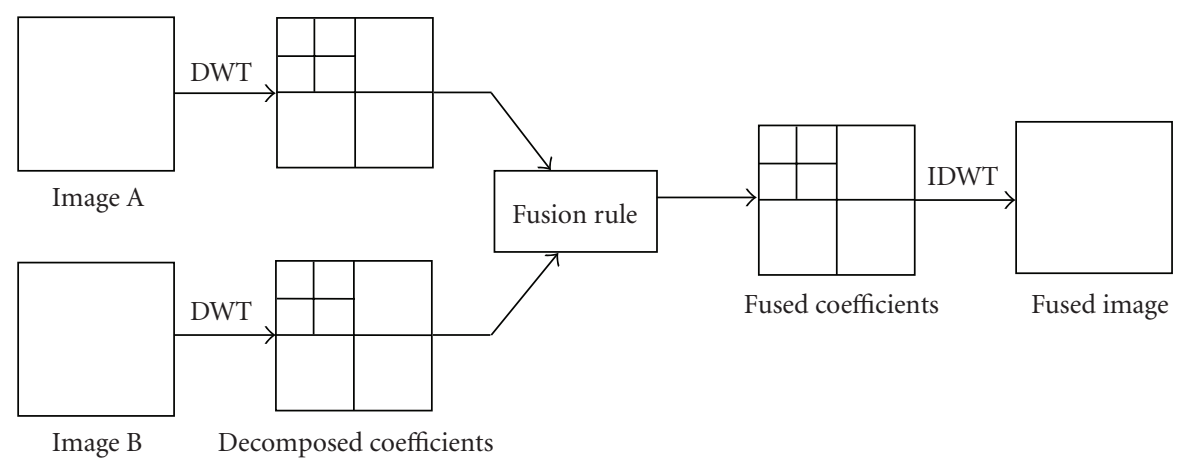

FIgURE 2: The image fusion scheme using the wavelet transform.

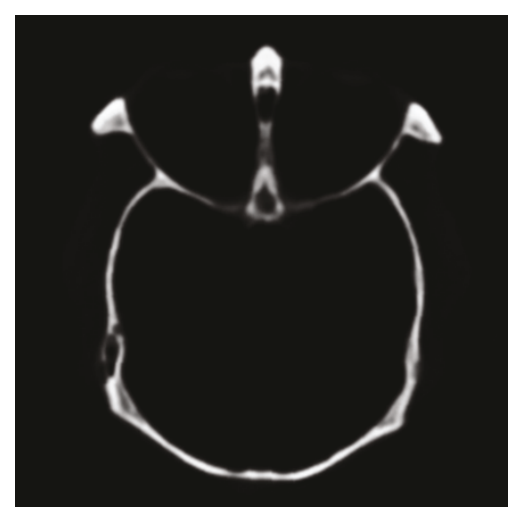

(a)

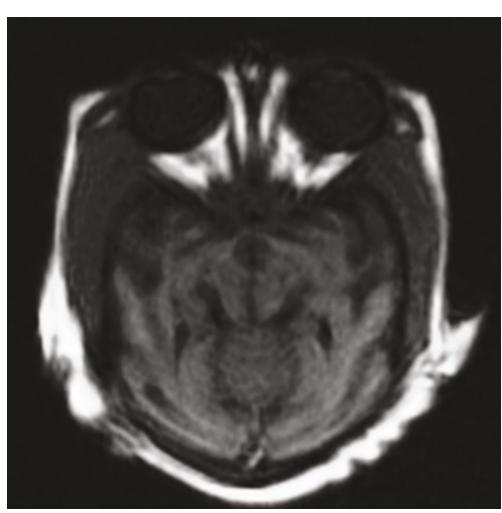

(b)

FIgURE 3: Original medical images to be fused. (a) Original CT image. (b) Original MR image.

The method can of course be easily extended to more than two images. Generally, an image $I$ has its multiscale decomposition (MSD) representation denoted $D_{I}$. Hence we will encounter $D_{X}, D_{Y}$, and $D_{Z}$. Let $p=(m, n, k, l)$ indicate the index corresponding to a particular MSD coefficient, where $m$ and $n$ indicate the spatial position in a given frequency band, $k$ is the decomposition level, and $l$ is the frequency band of the MSD representation. Therefore, $D_{I}(p)$ denote the MSD value of the corresponding coefficient at the position $(m, n)$ with decomposition level $k$ and frequency band $l$.

Since the low-frequency band is the original image at coarser resolution level, it can be considered as a smoothed and subsampled version of the original image. Therefore, most information of their source images is kept in the low-frequency band. Based on the pervious analysis, here for the low-frequency band, a fusion scheme which selects the highest local visibility is proposed. This approach is derived from [34] and is motivated by the fact that the HVS is sensitive to the contrast. Hence, this method can be likely to provide better details to the human observer. The fusion rule first calculates the window-based visibility of all coefficients in the low-frequency band. The visibility of wavelet coefficients is defined as

$$
\begin{aligned}
& \mathrm{VI}(p)= \frac{1}{w^{2}} \sum_{(i, j) \in B_{w}} \Lambda(\bar{D}(p)) \\
& \cdot \frac{|D(m+i, n+j, k, l)-\bar{D}(p)|}{\bar{D}(p)}, \\
& \bar{D}(p)= \frac{1}{w^{2}} \sum_{(i, j) \in B_{w}} D(m+i, n+j, k, l), \\
& \Lambda(\bar{D}(p))=\left(\frac{1}{\bar{D}(p)}\right)^{\alpha},
\end{aligned}
$$

where $B_{w}$ is a $w \times w$ block, $\Lambda(\bar{D}(p))$ is the weighting factor, $\mathrm{VI}(p)$ denote the visibility in the block, $\alpha$ is a visual constant obtained by perceptual experiment, and its range is from 0.6 to 0.7 [35]. After calculating the visibility of all the coefficients in the low-frequency band, the corresponding coefficients with higher magnitude of visibility are then chosen into the fused image as follows:

$$
D_{Z}(p)= \begin{cases}D_{X}(p), & \mathrm{VI}_{X}(p) \geq \mathrm{VI}_{Y}(p), \\ D_{Y}(p), & \mathrm{VI}_{X}(p)<\mathrm{VI}_{Y}(p) .\end{cases}
$$




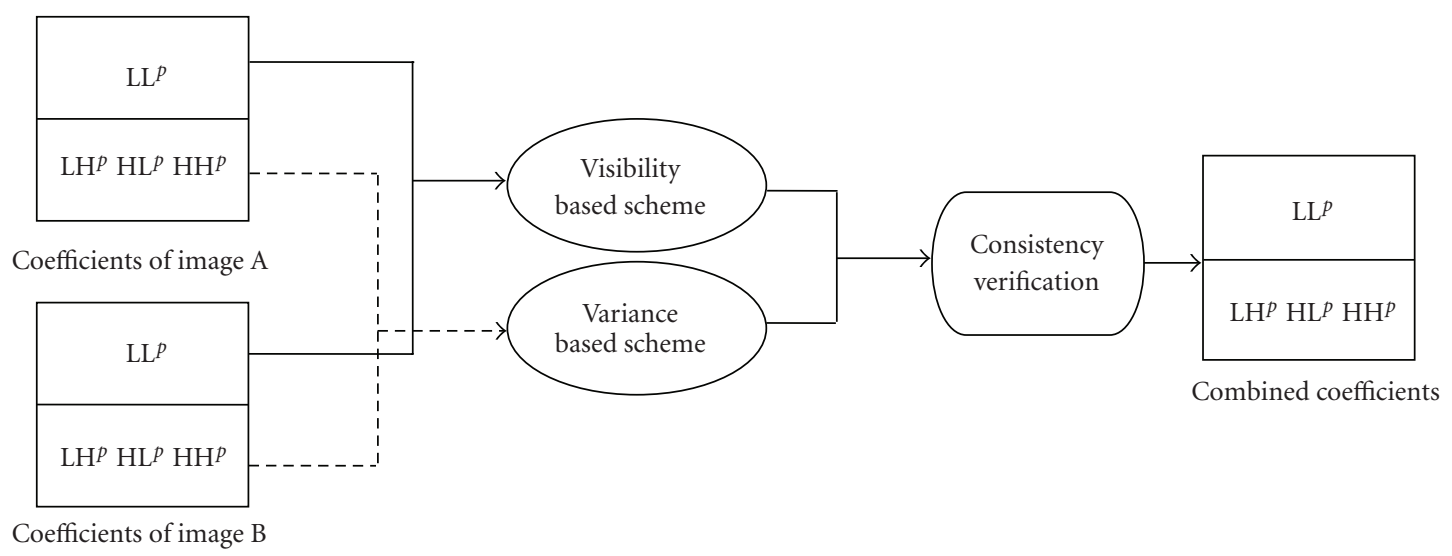

FIgURE 4: Schematic diagram of the proposed fusion rule.

3.2. High Frequency Bands Fusion. For the high-frequency bands, since the purpose of image fusion requires that the fused image must not discard any useful information contained in the source images and effectively preserve the details of input images such as edges, lines, and region boundaries, it is generally believed that the details of an image are mainly included in the high-frequency of the image. Therefore, it is important to find appropriate methods to merge the details of input images. The conventional selection of high-frequency coefficients only depend on their absolute value without taking any consideration of the neighboring coefficients. However, as we know a pixel in a image must have some relations with its neighboring pixels, which means that a MSD coefficient will also have relations with its neighboring MSD coefficients. In addition, according to characteristic of HVS [33] it is easy to find that for the high resolution region the human visual interest is concentrated on the detection of changes in contrast between regions on the edges separate these regions. Therefore, a good method for the high-frequency bands should produce large coefficients on those edges. Based on the above analysis, we propose a scheme by computing the variance in a neighborhood to select the high-frequency coefficients. The procedure can be formulated as follows:

$$
\begin{aligned}
\sigma_{I}(p)= & \frac{1}{S \times T} \sum_{s=-S / 2}^{S / 2} \sum_{t=-T / 2}^{T / 2}\left(D_{I}(m+s, n+t, k, l)\right. \\
& \left.-\operatorname{mean}_{I}(p)\right)^{2}, \\
\operatorname{mean}_{I}(p)= & \frac{1}{S \times T} \sum_{s=-S / 2}^{S / 2} \sum_{t=-T / 2}^{T / 2} D_{I}(m+s, n+t, k, l),
\end{aligned}
$$

where $S \times T$ is the neighboring size, and $\operatorname{mean}_{I}(p), \sigma_{I}(p)$ denote the mean value and variance value of the coefficients centered at $(m, n)$ in the window of $S \times T$, respectively. Then, the fusion scheme used for the high-frequency bands can be illustrated as follows:

$$
D_{Z}(p)= \begin{cases}D_{X}(p), & \sigma_{X}(p) \geq \sigma_{Y}(p), \\ D_{Y}(p), & \sigma_{X}(p)<\sigma_{Y}(p) .\end{cases}
$$

It is worthy to note again that the high-frequency bands referred here include the vertical, horizontal, and diagonal high-frequencies of the image, respectively. Therefore, the fusion process should be performed in all these domains.

3.3. Consistency Verification. As can be seen from above subsections all the coefficients of both low-frequency and high-frequency bands are selected by the maximum selection schemes, but as we know the maximum selection technique will be influent in case of noise. Furthermore, since we cope with the coefficients separately, this method cannot guarantee the homogeneity in the resultant fused image. Therefore, a consistency verification scheme is then performed, which can also ensure that the dominant features are incorporated as completely as possible into the fused image. The idea of this attempt is likely to be a majority filter. In this paper, we apply a window-based verification (WBV) to the coefficients in the composite MSD [18]. The WBV employs a small window centered at the current coefficient position. The WBV rule is that if the center composite MSD coefficient comes from image $X$, but the majority of the surrounding coefficients in the window come from image $Y$, then the center sample is changed to come from $Y$. In the implementation, this rule is applied to a binary decision map, and then it is followed by the application of a majority filter. The fused coefficients are finally obtained by the new 
binary decision map. This process can be formulated as follows:

$$
\begin{aligned}
& D_{X}^{\prime}(p)=\max _{w \in W}\left(\left|D_{X}(p, w)\right|\right), \\
& D_{Y}^{\prime}(p)=\max _{w \in W}\left(\left|D_{Y}(p, w)\right|\right), \\
& q_{X}(p)= \begin{cases}1, & D_{X}^{\prime}(p)>D_{Y}^{\prime}(p), \\
0, & \text { otherwise, }\end{cases} \\
& q_{Y}(p)= \begin{cases}1, & D_{Y}^{\prime}(p) \geq D_{X}^{\prime}(p), \\
0, & \text { otherwise, }\end{cases} \\
& q_{X}^{*}(p)= \begin{cases}1, & \sum q_{X}(p) \geq 5, \\
0, & \text { otherwise, }\end{cases} \\
& q_{Y}^{*}(p)=1-q_{X}^{*}(p), \\
& D_{Z}(p)=q_{X}^{*}(p) D_{X}(p)+q_{Y}^{*}(p) D_{Y}(p),
\end{aligned}
$$

where $W$ is a $3 \times 3$ window, and the value for the majority filter is set to 5 .

Through the above three procedures, the combined coefficients are then performed by an inverse wavelet transform, and the fused image can achieved consequently. Thus, the steps of our fusion approach in this paper can be briefly summarized as follows.

Step 1. Register the multimodal medical images.

Step 2. Decompose the images to 3-4 wavelet planes (resolution levels).

Step 3. The wavelet coefficients of the low-frequency are selected by (6) and (7), and the wavelet coefficients of the high-frequency are selected by (8).

Step 4. The coefficients of both the low-frequency and highfrequency are performed by the consistency verification of (10) and (18).

Step 5. Perform the inverse wavelet transform with the combined coefficients obtained from Step 4.

\section{Experimental Results and Analysis}

In this section, the application results of the proposed wavelet-based method for medical image fusion are presented. The performance of the proposed method is compared with those of pixel averaging method [36], the gradient pyramid method [14], and the conventional DWT method with maximum selection rule [20]. Since image registration is out of scope of this paper, like most of the literatures $[5,36]$, in all test cases we assume the source medical images to be in perfect registration. A thorough survey of image registration techniques can be referred to [37]. We use the Daubechies' db8, also with a decomposition level of 3 , as the wavelet-basis for DWT and the proposed method. A $3 \times 3$ window size for calculating the variance is considered in this paper, which has been proved to be more effective by many researchers $[38,39]$. We have carried out some comparisons on different values of the visual constant and found that the fusion result is insensitive to this parameter. Therefore, the parameter $\alpha$ is chosen to be 0.7 in this paper. Furthermore, we invited a radiologist (Associate Professor Xianjun Zeng, Department of the Medical Imaging, the First Affiliation Hospital of Nanchang University) to do subjective evaluation (visual assessment) of all the experiments.

To evaluate the performance of the proposed approach, tests were first realized on two simulated medical images as shown in Figure 5. An original T2-weighted MR image is shown in Figure 5(a), which served as the reference image here. Then two other images are generated by filtering the reference image with a Gaussian blurring process as $[5,11]$. Figure $5(\mathrm{~b})$ is the image blurred on the top, while Figure 5(c) is the image blurred on the bottom. Figures 5(d)$5(\mathrm{~g})$ are the fused results obtained by fusing Figure 5(b) and Figure 5(c) with the pixel averaging method, the gradient pyramid method, the DWT method, and the proposed method, respectively. The visual inspection of the fused image Figures $5(\mathrm{~d})-5(\mathrm{~g})$ was then carried out by the expert. However, results of his subjective evaluation reveal that through visual inspection it is difficult to find the difference of the four methods except that Figure 5(d) has a lower contrast. Therefore, a mutual information (MI) metric is employed here to objectively evaluate the performance of the four methods. This metric can indicate how much information the fused image conveys about the reference image [40]. Thus, the higher the MI, the better the result. The MI is defined as

$$
\operatorname{MI}\left(x_{R} ; x_{F}\right)=\sum_{u=1}^{L} \sum_{v=1}^{L} h_{R, F}(u, v) \log _{2} \frac{h_{R, F}(u, v)}{h_{R}(u) h_{F}(v)},
$$

where $x_{R}$ and $x_{F}$ denote the reference image and fused image, respectively, $h_{R, F}$ is the joint gray level histogram of $x_{R}$ and $x_{F}, h_{R}$ and $h_{F}$ are the normalized gray level histograms of $x_{R}$ and $x_{F}$, and $L$ is the number of bins. The MI values of the four different methods are calculated and shown in Figure 6. It can be seen from Figure 6 that the MI value of the proposed method is the largest in the four methods, and the MI value of the pixel averaging method is the smallest. The results presented in this example can demonstrate that our approach can fuse the medical image while retaining much more information than that of the other three methods.

The second example is the frequently used normal CT and MR images as shown in Figure 3. The experimental results of the above four method are displayed in Figures $7(\mathrm{a})-7(\mathrm{~d})$, respectively. Compared with the original CT and MR images in Figure 3, it is easy to find that with all the methods the fused image now contains both the bones information and tissues information, which cannot be seen in the separate CT or MR image. However, after careful manual inspection of Figures 7(a)-7(d) by our expert, he indicated that the fused result of the proposed method is the best in the four methods because the information of bones and tissues is clearer than other three methods, while 


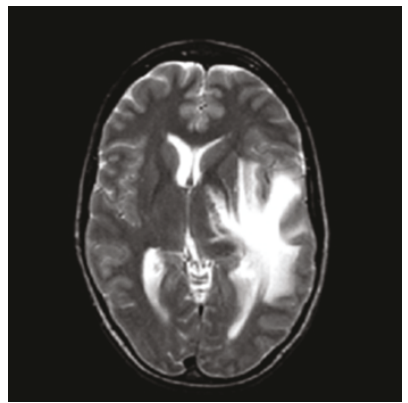

(a)

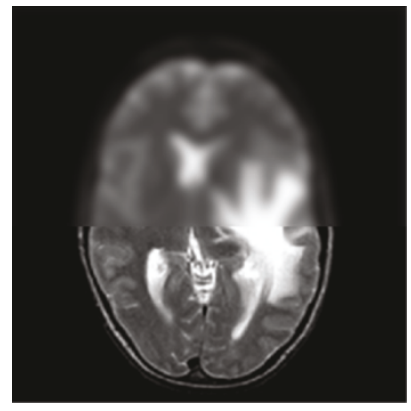

(b)

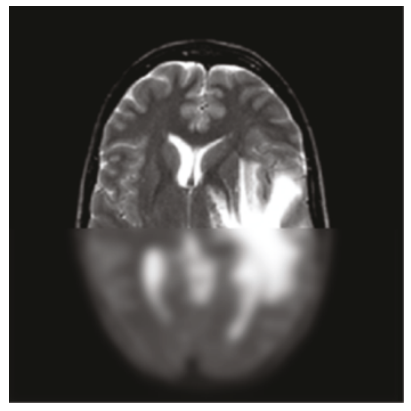

(c)

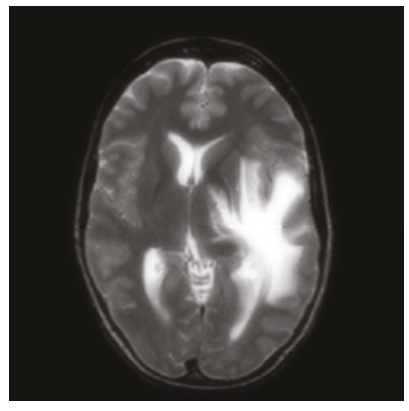

(d)

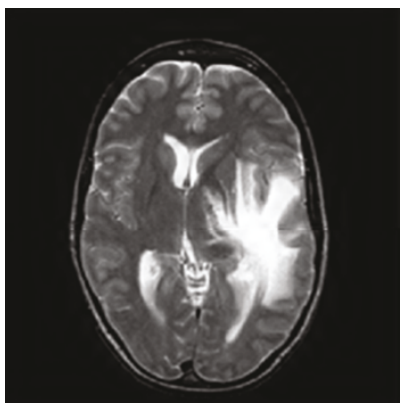

(f)

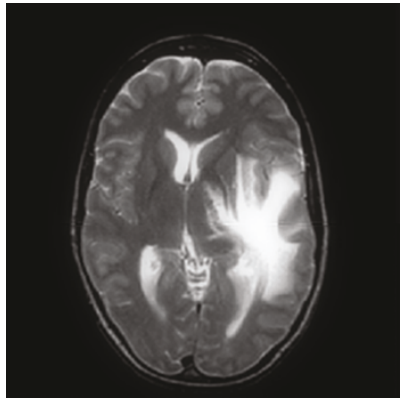

(e)

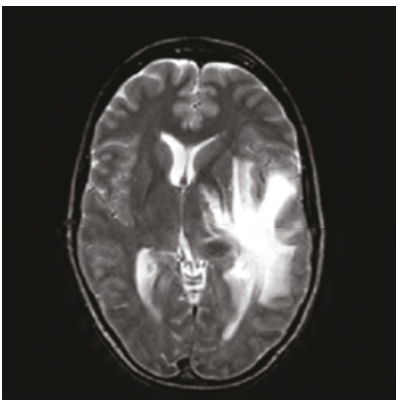

(g)

FIGURE 5: Medical image fusion with the simulated pair from a T2-weighted MR image. (a) The original medical image (reference image or ground truth); (b) image blurred on the top; (c) image blurred on the bottom; (d) fused image by pixel averaging; (e) fused image by gradient pyramid; (f) fused image by DWT; (g) fused image by the proposed method.

TABLE 1: Quantitative evaluation results of the four different fusion methods in Figure 7.

\begin{tabular}{lcccc}
\hline Fusion methods & Standard deviation & Average gradient & Information entropy & Cross entropy \\
\hline Pixel averaging & 34.8582 & 3.8985 & 5.7602 & 2.0768 \\
Gradient pyramid & 38.7825 & 5.2708 & 6.1359 & 1.7799 \\
DWT & 41.1598 & 6.7343 & 6.1781 & 1.9428 \\
Proposed method & 57.9787 & 7.5005 & 6.7295 & 0.9626 \\
\hline
\end{tabular}

TABLE 2: Quantitative evaluation results of the four different fusion methods in Figure 8.

\begin{tabular}{lcccc}
\hline Fusion methods & Standard deviation & Average gradient & Information entropy & Cross entropy \\
\hline Pixel averaging & 45.5339 & 6.4783 & 5.9594 & 2.8611 \\
Gradient pyramid & 46.2360 & 8.6048 & 6.5275 & 2.9881 \\
DWT & 50.8397 & 10.1953 & 6.0989 & 2.7748 \\
Proposed method & 68.8098 & 10.4994 & 6.5681 & 1.8884 \\
\hline
\end{tabular}




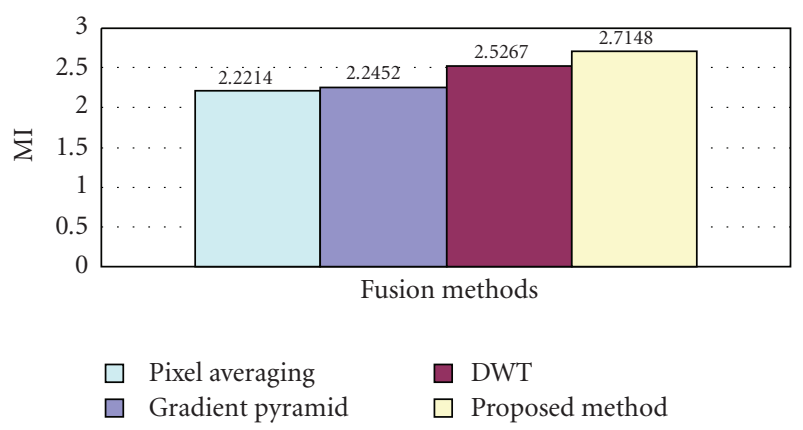

Figure 6: MI values of the four different methods.

the result of the pixel averaging is the worst because the information of bones and tissues is very blurry or fuzzy, and the result of gradient pyramid is almost identical to that of DWT method. However, just as in [41] the subjective evaluation depends on the expert's experience and some uncertainty is involved because this measure has no rigorous mathematical models and is mainly visual. Considering the drawbacks of the subjective quality evaluation method, quantitative evaluation of the quality of the fused images is thus needed, which will be more objective than the visual inspection. In addition, based on the requirements of fusion algorithm [42], when we evaluate the performance of the fusion technique, we must pay attention to that (1) it should preserve all relevant information of the input images in the fused image (pattern conservation); (2) it should minimise any artifacts or inconsistence in the fused image. Only in this case, we can accurately and comprehensively explain which fusion method is more effective.

Therefore, in order to better evaluate the above fusion methods, quantitative assessment of the performance of the four methods is then carried out. However, as we know actually for image fusion it is often hard to get the ideal or reference composite image; so the above MI metric cannot be used here. Consequently, four other evaluation criteria are then introduced and employed in this paper $[41,43]$.

(i) Standard Deviation. The standard deviation of an image with size of $M \times N$ is defined as

$$
\hat{\sigma}=\left(\frac{1}{M \times N} \sum_{m=1}^{M} \sum_{n=1}^{N}(f(m, n)-\hat{\mu})^{2}\right)^{1 / 2},
$$

where $f(m, n)$ is the pixel value of the fused image at the position $(m, n), \hat{\mu}$ is the mean value of the image. The standard deviation is the most common measure of statistical dispersion, which can be used to evaluate how widely spread the gray values in an image. So, the larger the standard deviation, the better the result. (ii) Average Gradient. The average gradient of an image with size of $M \times N$ is defined as:

$$
\begin{aligned}
\operatorname{Avg}= & \frac{1}{(M-1) \times(N-1)} \\
& \times \sum_{m=1}^{M-1} \sum_{n=1}^{N-1} \sqrt{\left[\left(\frac{\partial f(m, n)}{\partial m}\right)^{2}+\left(\frac{\partial f(m, n)}{\partial n}\right)^{2}\right] / 2},
\end{aligned}
$$

where $f(m, n)$ is the same meaning as in the standard deviation. The average gradient reflects the clarity of the fused image. It is used to measure the spatial resolution of the fused image; that is, larger average gradient means a higher resolution.

(iii) Information Entropy. The formulation of the classical information entropy of an image is defined as

$$
H=-\sum_{l=0}^{L-1} P_{l} \log _{2} P_{l}
$$

where $L$ is the number of gray level, and $P_{l}$ equals the ratio between the number of pixels whose gray value is $l(0 \leq l \leq$ $L-1)$ and the total pixel number contained in the image. The information entropy measures the richness of information in an image. Thus, the higher the entropy, the better the performance.

(iv) Cross Entropy (CE). The cross entropy is used to measure the difference between the source images and the fused image. Small value corresponds to good fusion result obtained:

$$
\mathrm{CE}=\sum_{l=0}^{L-1} P_{l} \log _{2} \frac{P_{l}}{Q_{l}},
$$

where $P_{l}$ and $Q_{l}$ denote the gray level histogram of the source image and fused image, respectively.

The above four evaluation criteria are then applied to evaluate the four fusion methods in Figure 7, and the detailed quantitative results are given in Table 1. From Table 1, we can observe that the values of several quality indices such as the standard deviation, average gradient, and information entropy of the proposed method are larger than those of pixel averaging, gradient pyramid, and DWT methods. For instance, the average gradient of the proposed method is 7.5005 , while the corresponding values of other three methods are $3.8985,5.2708$, and 6.7343 , respectively. These three largest values presented here can indicate that with the proposed method the fused image can get higher spatial resolution and retain much more image information. The last column of Table 1 shows the values of the cross entropy of the four methods. By comparison, it can be seen that the cross entropy value of the proposed method is the smallest in the four methods. This means that with the proposed method the fused images have less difference to the source 


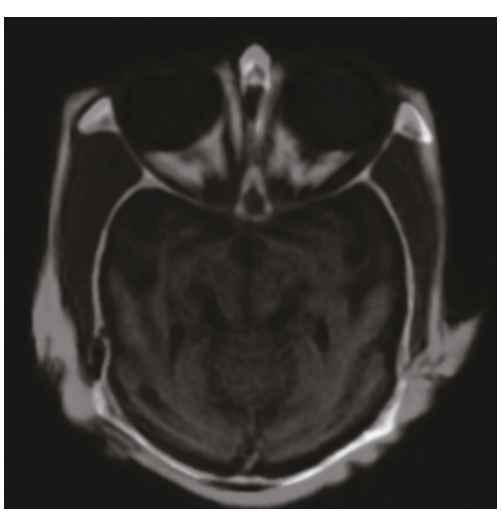

(a)

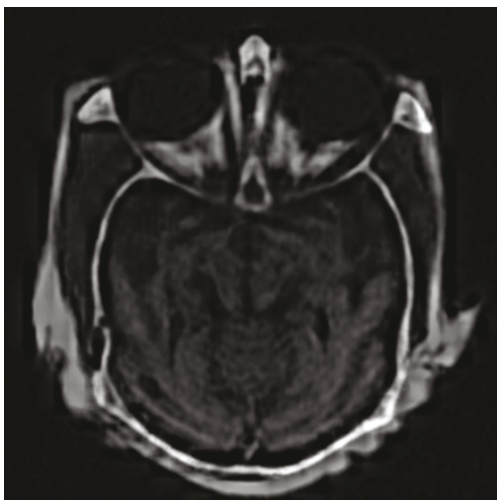

(c)

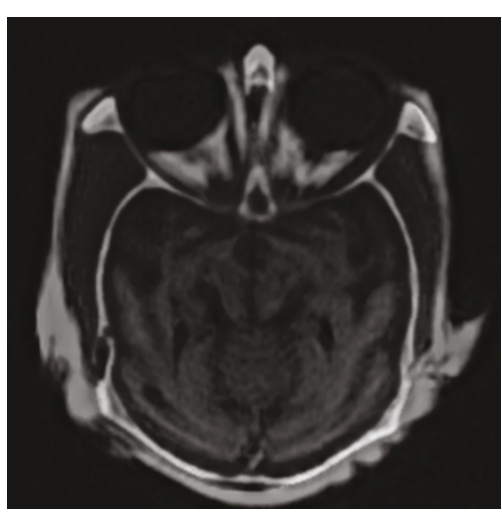

(b)

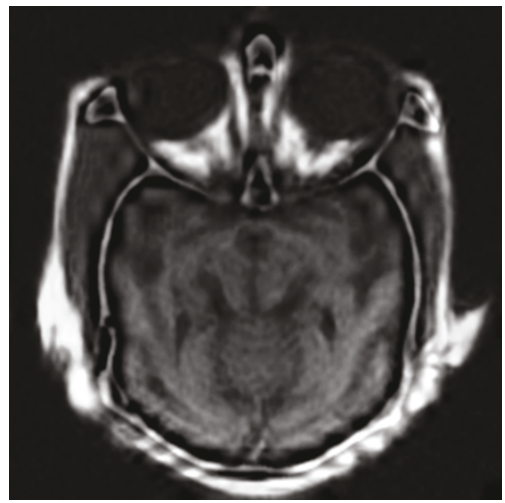

(d)

FIGURE 7: Fusion results of the CT and MR images with different methods. (a) Fused image by pixel averaging; (b) fused image by gradient pyramid; (c) fused image by DWT; (d) fused image by the proposed method.

images than those of other three methods. From Table 1, we can also find that the performance of the pixel averaging is the worst in the four methods; the performance of the DWT method is somewhat superior to that of gradient pyramid method.

The last examples are two medical images, one is a T1weighted MR image, and another is an MRA image with some illness as shown in Figures 8 (a) and 8(b), respectively. From these two images, it can be seen that in the T1-weighted MR image, the soft tissue is clear and easy to recognize, but the illness medical information as shown in the marked ellipse area of Figure 8(b) has been lost. On the contrary, although the MRA image contains the illness information, the soft tissues in it are very difficult to distinguish due to its lower spatial resolution. Therefore, in order to support entire and accurate medical information for doctor's analysis and diagnosis, the fusion of the two images is required. The four methods mentioned above are then used to fuse those two images, and their corresponding results are displayed in Figures $8(\mathrm{c})-8(\mathrm{f})$, respectively. As can be seen, with all the methods the fused images now appear to preserve the overall regions of interest (ROI) presented in the two images. However, by our expert subjectively observing, he claimed that the fused result of the proposed method is more clearly and has a higher contrast than that of the other three methods, but it is hard to discriminate the three fused images of the pixel averaging, the gradient pyramid, and the DWT methods in this visual case. Hence, in order to better evaluate the performance of the four methods, quantitative assessments are also carried out with the above evaluation criteria, and their corresponding results are listed in Table 2. From Table 2, it is easy to see, just as Table 1, that the performance of the proposed method is the best in the four methods because it not only has the highest values of the standard deviation, average gradient, and information entropy, respectively, but also has the lowest value of the cross entropy. Therefore, based on these two experimental results on the real medical images presented here and according to the requirements of fusion method mentioned above, we can conclude that all the quantitative evaluations are basically corresponding to the doctor's visual effects, and the proposed wavelet-based fusion method performs better than the other three existing fusion methods.

\section{Conclusions}

The fusion of multimodal medical images plays an important role in many clinical applications for they can support more accurate information than any individual source image. This paper presents a novel wavelet-based approach for medical image fusion, which consists of three steps. In the first 


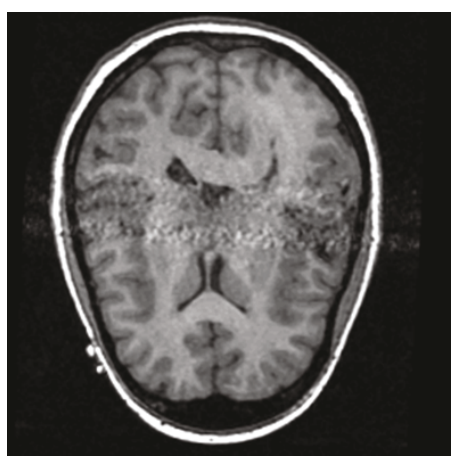

(a)

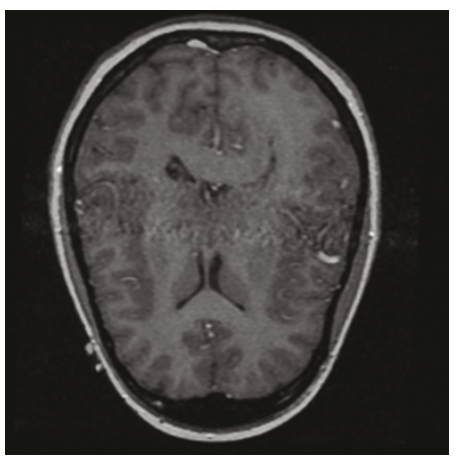

(c)

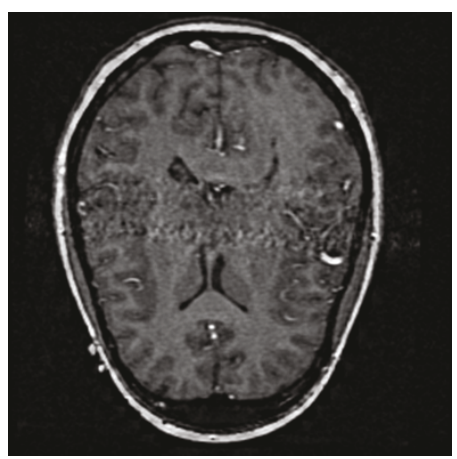

(e)

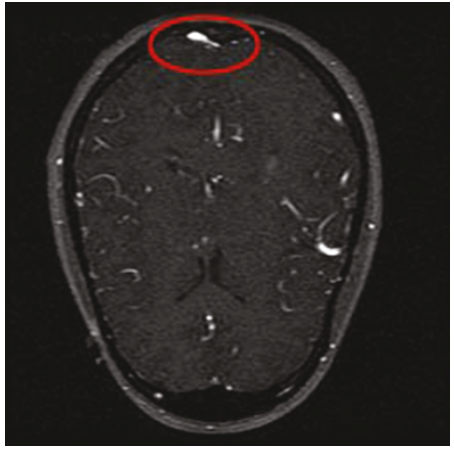

(b)

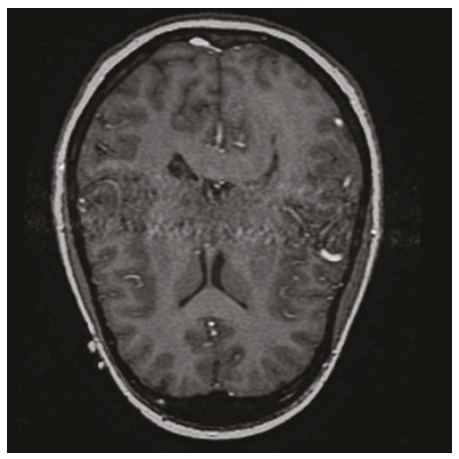

(d)

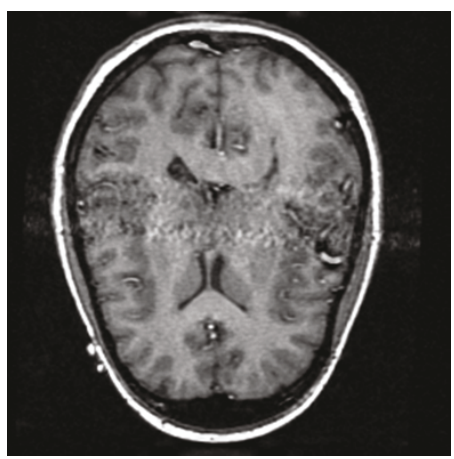

(f)

FIGURE 8: Fusion results of the T1-weighted MR and MRA images with different methods. (a) Original T1-weighted MR image; (b) original MRA image; (c) fused image by pixel averaging; (d) fused image by gradient pyramid; (e) fused image by DWT; (f) fused image by the proposed method.

step, the medical images to be fused are decomposed into subimages by wavelet transform. In the second step, after considering the characteristics of HVS and the physical meaning of the wavelet coefficients, the coefficients of the low-frequency band and high-frequency bands are performed with different fusion strategies: the former is selected using a maximum visibility scheme, and the latter is selected by a maximum local variance rule. In order to improve the quality of the resultant image, all the combined coefficients are then performed by a window based consistency verification. In the last step, the fused image is constructed by the inverse wavelet transform with the composite coefficients. The performance of the proposed method is qualitatively and quantitatively compared with some existing fusion approaches. Experimental results show that the proposed method can preserve more useful information in the fused image with higher spatial resolution and less difference to the source images.

\section{Acknowledgments}

This work was supported by the National Natural Science Foundation of China under the grant no. 60963012, by the Ministry of Education, Science Technology (MEST) and Korea Industrial Technology Foundation (KOTEF) though the Humana Resource Training Project for Regional Innovation, by the second stage of Brain Korea 21, by the China Postdoctoral Special Science Foundation funded project under the grant no. 200902614, and by the Science and 
Technology Research Project of the Education Department of Jiangxi Province under the grants no. GJJ10125 and no. GJJ09287. The authors also thank the anonymous referees for their valuable suggestions.

\section{References}

[1] F. Maes, D. Vandermeulen, and P. Suetens, "Medical image registration using mutual information," Proceedings of the IEEE, vol. 91, no. 10, pp. 1699-1721, 2003.

[2] V. Barra and J.-Y. Boire, "A general framework for the fusion of anatomical and functional medical images," NeuroImage, vol. 13, no. 3, pp. 410-424, 2001.

[3] Y.-M. Zhu and S. M. Cochoff, "An object-oriented framework for medical image registration, fusion, and visualization," Computer Methods and Programs in Biomedicine, vol. 82, no. 3, pp. 258-267, 2006.

[4] V. S. Petrovic and C. S. Xydeas, "Gradient-based multiresolution image fusion," IEEE Transactions on Image Processing, vol. 13, no. 2, pp. 228-237, 2004.

[5] Z. Zhang and R. S. Blum, "A categorization of multiscaledecomposition-based image fusion schemes with a performance study for a digital camera application," Proceedings of the IEEE, vol. 87, no. 8, pp. 1315-1326, 1999.

[6] Y. Wang and B. Lohmann, "Multisensor image fusion: concept, method and applications," Tech. Rep., Institute of Automatic Technology, University of Bremen, Bremen, Germany, 2000.

[7] S. T. Shivappa, B. D. Rao, and M. M. Trivedi, "An iterative decoding algorithm for fusion of multimodal information," EURASIP Journal on Advances in Signal Processing, vol. 2008, Article ID 478396, 10 pages, 2008.

[8] R. Redondo, F. Sroubek, S. Fischer, and G. Cristobal, "Multifocus image fusion using the log-Gabor transform and a Multisize Windows technique," Information Fusion, vol. 10, no. 2, pp. 163-171, 2009.

[9] S. Li and B. Yang, "Multifocus image fusion using region segmentation and spatial frequency," Image and Vision Computing, vol. 26, no. 7, pp. 971-979, 2008.

[10] P. S. Pradhan, R. L. King, N. H. Younan, and D. W. Holcomb, "Estimation of the number of decomposition levels for a wavelet-based multiresolution multisensor image fusion," IEEE Transactions on Geoscience and Remote Sensing, vol. 44, no. 12, pp. 3674-3686, 2006.

[11] S. Li, J. T. Kwok, and Y. Wang, "Multifocus image fusion using artificial neural networks," Pattern Recognition Letters, vol. 23, no. 8, pp. 985-997, 2002.

[12] J. Yang and R. S. Blum, "A statistical signal processing approach to image fusion for conceled weapon detection," in Proceedings of the IEEE International Conference on Image Processing, vol. 1, pp. 513-516, 2002.

[13] P. J. Burt and E. H. Adelson, "The Laplacian pyramid as a compact image code," IEEE Transactions on Communications, vol. 31, no. 4, pp. 532-540, 1983.

[14] P. J. Burt and R. J. Kolczynski, "Enhanced image capture through fusion," in Proceedings of the 4th IEEE International Conference on Computer Vision (ICCV'93), pp. 173-182, 1993.

[15] A. Toet, J. J. van Ruyven, and J. M. Valeton, "Merging thermal and visual images by a contrast pyramid," Optical Engineering, vol. 28, no. 7, pp. 789-792, 1989.

[16] A. Toet, "Image fusion by a ration of low-pass pyramid," Pattern Recognition Letters, vol. 9, no. 4, pp. 245-253, 1989.

[17] A. Toet, "A morphological pyramidal image decomposition," Pattern Recognition Letters, vol. 9, no. 4, pp. 255-261, 1989.
[18] H. Li, B. S. Manjunath, and S. K. Mitra, "Multisensor image fusion using the wavelet transform," Graphical Models and Image Processing, vol. 57, no. 3, pp. 235-245, 1995.

[19] G. K. Matsopoulos and S. Marshall, "Application of morphological pyramids: fusion of MR and CT phantoms," Journal of Visual Communication and Image Representation, vol. 6, no. 2, pp. 196-207, 1995.

[20] L. J. Chipman, T. M. Orr, and L. N. Graham, "Wavelets and image fusion," in Proceedings of the IEEE International Conference on Image Processing, vol. 3, pp. 248-251, 1995.

[21] T. Pu and G. Ni, "Contrast-based image fusion using the discrete wavelet transform," Optical Engineering, vol. 39, no. 8, pp. 2075-2082, 2000.

[22] H. Ma, C. Y. Jia, and S. Liu, "Multisource image fusion based on wavelet transform," International Journal of Information Technology, vol. 11, no. 7, pp. 81-91, 2005.

[23] F. W. Acerbi-Junior, J. G. P. W. Clevers, and M. E. Schaepman, "The assessment of multi-sensor image fusion using wavelet transforms for mapping the Brazilian Savanna," International Journal of Applied Earth Observation and Geoinformation, vol. 8, no. 4, pp. 278-288, 2006.

[24] G. Pajares and J. M. D. L. Cruz, "A wavelet-based image fusion tutorial," Pattern Recognition, vol. 37, no. 9, pp. 1855-1872, 2004.

[25] K. Amolins, Y. Zhang, and P. Dare, "Wavelet based image fusion techniques - an introduction, review and comparison," ISPRS Journal of Photogrammetry \& Remote Sensing, vol. 62, no. 4, pp. 249-263, 2007.

[26] L. F. Yu, D. L. Zu, W. D. Wang, and S. L. Bao, "Multi-modality medical image fusion based on wavelet analysis and quality evaluation," Journal of Systems Engineering and Electronics, vol. 12, no. 1, pp. 42-48, 2001.

[27] G. H. Qu, D. L. Zhang, and P. F. Yan, "Medical image fusion by wavelet transform modulus maxima," Optics Express, vol. 9, no. 4, pp. 184-190, 2001.

[28] S. Garg, K. U. Kiran, R. Mohan, and U. S. Tiwary, "Multilevel medical image fusion using segmented image by level set evolution with region competition," in Proceedings of the 27th Annual International Conference of the IEEE Engineering in Medicine and Biology (EMBS '06), pp. 7680-7683, 2006.

[29] S. L. Cheng, J. M. He, and Z. W. Lv, "Medical image of PET/CT weighted fusion based on wavelet transform," in Proceedings of the 2nd International Conference on Bioinformatics and Biomedical Engineering (iCBBE '08), pp. 2523-2525, 2008.

[30] S. G. Mallat, "A theory for multiresolution signal decomposition: the wavelet representation," IEEE Transactions on Pattern Analysis and Machine Intelligence, vol. 11, no. 7, pp. 674-693, 1989.

[31] S. G. Nikolov, P. Hill, D. R. Bull, and C. N. Canagarajah, "Wavelets for image fusion," in Wavelets in Signal and Image Analysis, Computational Imaging and Vision Series, pp. 213-244, Kluwer Academic Publishers, Dodrecht, The Netherlands, 2001.

[32] A. S. Lewis and G. Knowles, "Image compression using the 2D wavelet transform," IEEE Transactions on Image Processing, vol. 1, no. 2, pp. 244-250, 1992.

[33] M. Barni, F. Bartolini, and A. Piva, "Improved wavelet-based watermarking through pixel-wise masking," IEEE Transactions on Image Processing, vol. 10, no. 5, pp. 783-791, 2001.

[34] J. W. Huang, Q. S. Yun, and X. H. Dai, “A segmentation-based image coding algorithm using the features of human vision system," Journal of Image and Graphics, vol. 4, no. 5, pp. 400404, 1999. 
[35] A. B. Watson, "Efficiency of a model human image code," Journal of the Optical Society of America. A, vol. 4, no. 12, pp. 2401-2417, 1987.

[36] N. Mitianoudis and T. Stathaki, "Pixel-based and region-based image fusion schemes using ICA bases," Information Fusion, vol. 8, no. 2, pp. 131-142, 2007.

[37] B. Zitova and J. Flusser, "Image registration methods: a survey," Image and Vision Computing, vol. 21, no. 11, pp. 9771000, 2003.

[38] G.-X. Liu and W.-H. Yang, "A wavelet-decomposition-based image fusion scheme and its performance evaluation," Acta Automatica Sinica, vol. 28, no. 6, pp. 927-934, 2002.

[39] M. Li, X. Y. Zhang, and J. Mao, "Neighboring region variance weighted mean image fusion based on wavelet transform," Foreign Electronic Measurement Technology, vol. 27, no. 1, pp. 5-6, 2008.

[40] G. Piella, "A general framework for multiresolution image fusion: from pixels to regions," Information Fusion, vol. 4, no. 4, pp. 259-280, 2003.

[41] W. Z. Shi, C. Q. Zhu, Y. Tian, and J. Nichol, "Wavelet-based image fusion and quality assessment," International Journal of Applied Earth Observation and Geoinformation, vol. 6, no. 3-4, pp. 241-251, 2005.

[42] Y. F. Zheng, E. A. Essock, B. C. Hansen, and A. M. Haun, "A new metric based on extended spatial frequency and its application to DWT based fusion algorithms," Information Fusion, vol. 8, no. 2, pp. 177-192, 2007.

[43] M. Li, W. Cai, and Z. Tan, "A region-based multi-sensor image fusion scheme using pulse-coupled neural network," Pattern Recognition Letters, vol. 27, no. 16, pp. 1948-1956, 2006. 